\title{
Characteristics associated with genetic counseling referral and $B R C A 1 / 2$ testing among women in a large integrated health system
}

\author{
Cecelia A. Bellcross, PhD, MS1, Lucy A. Peipins, PhD², Frances A. McCarty, PhD, \\ Juan L. Rodriguez, MS, MPH², Nikki A. Hawkins, PhD², Sharon Hensley Alford, MPH, PhD 4 \\ and Steven Leadbetter, MS²
}

\begin{abstract}
Background: Evidence shows underutilization of cancer genetics services. To explore the reasons behind this underutilization, this study evaluated characteristics of women who were referred for genetic counseling and/or had undergone BRCA1/2 testing.

Methods: An ovarian cancer risk perception study stratified 16,720 eligible women from the Henry Ford Health System into average-, elevated-, and high-risk groups based on family history. We randomly selected 3,307 subjects and interviewed 2,524 of them $(76.3 \%$ response rate).
\end{abstract}

Results: Among the average-, elevated-, and high-risk groups, 2.3, 10.1 , and $20.2 \%$, respectively, reported genetic counseling referrals, and $0.8,3.3$, and $9.5 \%$, respectively, reported having undergone $B R C A$ testing. Personal breast cancer history, high risk, and perceived ovarian cancer risk were associated with both referral and testing.
Discussion of family history with a doctor predicted counseling referral, whereas belief that family history influenced risk was the strongest $B R C A$ testing predictor. Women perceiving their cancer risk as much higher than other women their age were twice as likely (95\% confidence interval: 2.0-9.6) to report genetic counseling referral.

Conclusion: In a health system with ready access to cancer genetic counseling and $B R C A$ testing, women who were at high risk underutilized these services. There were strong associations between perceived ovarian cancer risk and genetic counseling referral, and between a belief that family history influenced risk and $B R C A$ testing.

Genet Med advance online publication 19 June 2014

Key Words: $B R C A 1$ and $B R C A 2$ genes; family history; genetic counseling; genetic testing; hereditary breast and ovarian cancer syndrome

\section{INTRODUCTION}

Hereditary breast and ovarian cancer syndrome (HBOC), which is associated with the BRCA1 or BRCA2 (BRCA1/2) genes, accounts for an estimated 2-7\% of breast cancers and $10-15 \%$ of ovarian cancers. Approximately 1 in 400 individuals in the general population and 1 in 40 individuals of Ashkenazi Jewish descent have a mutation in $B R C A 1 / 2 \cdot{ }^{1-3}$ Lifetime risks for breast and ovarian cancer in women who carry $B R C A 1 / 2$ mutations range from 40 to $80 \%$ and from 10 to $40 \%$, respectively. ${ }^{4}$ Firstdegree relatives (male and female) of known mutation carriers have a $50 \%$ chance of inheriting the familial mutation. Earlier and more frequent cancer screening, use of breast magnetic resonance imaging, and surgical risk-reducing options, such as prophylactic mastectomy and bilateral salpingo-oophorectomy, have shown clear potential for reducing cancer incidence and mortality and improving the likelihood of early detection for BRCA1/2 mutation carriers. ${ }^{5-7}$

Many groups, such as the National Comprehensive Cancer Network, the US Preventive Services Task Force, the American College of Obstetrics and Gynecology, and the National Society of Genetic Counselors, support the identification and appropriate management of $B R C A$ mutation carriers and have published related guidelines. ${ }^{8-11}$ Moreover, a substantial proportion of health insurers cover the cost of $B R C A$ testing for at-risk individuals, although genetic counseling is less consistently reimbursed. ${ }^{12}$ Despite the growing support of the medical and payer communities, and the availability of $B R C A$ testing since 1996, it is estimated that most individuals who carry $B R C A$ mutations remain unidentified. ${ }^{13}$

Although there is great interest in genetic testing among the general public, ${ }^{14}$ the low uptake of genetic counseling and testing for HBOC might be explained in part by barriers occurring at the patient, provider, and system levels. ${ }^{15}$ Factors known to pose challenges to both referral and utilization of cancer risk counseling and testing for BRCA1/2 mutations include cost and insurance coverage, provider awareness, race/ethnicity, concerns regarding insurance discrimination, and psychosocial barriers. ${ }^{16-18}$ The decision to undergo genetic counseling and testing is multifaceted and may be spurred by a desire to understand one's own risk for cancer as well as risks for one's

\footnotetext{
${ }^{1}$ Department of Human Genetics, Emory University School of Medicine, Atlanta, Georgia, USA; ${ }^{2}$ Epidemiology and Applied Research Branch, Division of Cancer Prevention and Control, Centers for Disease Control and Prevention, Atlanta, Georgia, USA; ${ }^{3}$ National Center for Health Statistics, Office of Public Health Science Services, Centers for Disease Control and Prevention, Hyattsville, Maryland, USA; ${ }^{4}$ Josephine Ford Cancer Center, Henry Ford Health System, Detroit, Michigan, USA. Correspondence: Cecelia A. Bellcross (cbellcr@emory.edu)
} 
children and other relatives. ${ }^{19,20}$ In addition, a personal experience with breast or ovarian cancer ${ }^{19}$ and a strong family history of cancer ${ }^{21,22}$ are significant motivators to seek cancer genetics services. A heightened perception of personal vulnerability to cancer, often resulting from an overestimation of personal risk, ${ }^{23,24}$ might also influence patient participation in genetic counseling and testing for HBOC.

Our study evaluates the frequency of genetic counseling referral and BRCA1/2 testing among women at average, elevated, and high risk based on family history of breast and ovarian cancer, as well as Ashkenazi ancestry, from a large integrated health system with both coverage for and ready access to these services. This analysis also explores individual-level characteristics that may affect genetic counseling referral and BRCA1/2 testing, and examines the influence of key aspects of family cancer history, sociodemographic characteristics, and risk perception factors on the uptake of cancer genetics services.

\section{MATERIALS AND METHODS}

\section{Study population, eligibility criteria, and design}

Data were obtained from a study conducted by the Centers for Disease Control and Prevention that evaluated ovarian cancer risk perception and screening among women in the Henry Ford Health System (HFHS), Detroit, Michigan. Detailed information on survey design and implementation has been previously published. ${ }^{25}$ Briefly, computer-assisted telephone interviews were conducted between 16 January and 13 December 2008 among eligible women $\geq 30$ years of age, with no previous history of ovarian cancer or bilateral oophorectomy.

For random sampling purposes, we used responses from the eligibility screener to stratify women into three risk groups based on the number of first- and second-degree relatives with breast or ovarian cancer and personal history of breast cancer. HFHS administrative data identified 55,887 potential study participants; of these 20,483 underwent eligibility screening (36.7\%) and $16,720(81.6 \%)$ were deemed eligible. The computer-assisted telephone interview system randomly selected 3,307 women for participation in the study. Women in the elevated- and high-risk groups based on the eligibility screener were oversampled, and subsequent analyses were weighted. A total of 2,524 women gave consent and were interviewed, for a response rate of $76.3 \%$.

The interview questions addressed detailed information about family history of cancer (including breast and ovarian) among first- and second-degree relatives in both maternal and paternal lineages, as well as age of diagnosis and the occurrence of bilateral breast cancer and male breast cancer, and Jewish ancestry. This additional information was used to assign women more accurately to average-, elevated-, and high-risk categories with regard to risk for breast and/or ovarian cancer and the likelihood of a BRCA1/2 mutation. The cancer family history was further classified by lineage as maternal, paternal, both, or no family history.

Table 1 illustrates the minimum characteristics used to assign subjects to the elevated- and high-risk groups, whereas those subjects not meeting any of these characteristics were considered
Table 1 Personal and family cancer history features used to assign participants to high- or elevated-risk categories

\begin{tabular}{|c|c|c|}
\hline Category ${ }^{a}$ & High risk & Elevated risk \\
\hline Non-Jewish & $\begin{array}{l}\text { - } 2 \text { FD relatives with br ca, } \\
1 \text { dx } \leq \text { age } 50 \\
\text { - } 3 \text { Or more FD and/or SD } \\
\text { relatives with br ca dx at } \\
\text { any age } \\
\text { - } 1 \text { FD relative with bilateral } \\
\text { br ca } \\
\text { - } 1 \text { FD or SD relative with } \\
\text { both br ca and ov ca } \\
\text { - } 1 \text { FD or SD male relative } \\
\text { with br ca } \\
\text { - Personal hx br ca dx } \\
\leq \text { age } 50 \\
\text { - } 2 \text { FD and/or SD relatives } \\
\text { with ov ca } \\
\text { - } 1 \text { FD and } 1 \text { SD relative with } \\
\text { br ca dx } \leq \text { age } 50 \\
\text { - } 2 \text { Paternal SD relatives } \\
\text { with br ca dx } \leq \text { age } 50\end{array}$ & $\begin{array}{l}\text { - } 1 \text { FD relative with } \\
\text { ov ca } \\
\text { - } 2 \text { Maternal SD } \\
\text { relatives with br ca dx } \\
\leq \text { age } 50 \\
\text { - Personal hx br ca dx } \\
>\text { age } 50 \\
\text { - } 2 \mathrm{SD} \text { relatives with br } \\
\text { ca dx > age } 50 \\
\text { - } 1 \mathrm{FD} \text { and } 1 \mathrm{SD} \text { relative } \\
\text { with br ca, } 1 \mathrm{dx} \leq \\
\text { age } 50 \\
\text { - } 1 \mathrm{FD} \text { relative with br } \\
\text { ca dx } \leq \text { age } 50\end{array}$ \\
\hline Jewish & $\begin{array}{l}\text { - } 1 \text { FD relative with br ca } \\
\text { - } 1 \text { SD relative with br ca dx } \\
\leq \text { age } 50 \\
\text { - } 1 \text { FD or SD relative with } \\
\text { ov ca } \\
\text { - } 2 \text { SD relatives with br ca } \\
\text { or } 1 \text { with br ca and } 1 \text { with } \\
\text { ov ca } \\
\text { - FD or SD male relative with } \\
\text { br ca } \\
\text { - Personal hx br ca dx any } \\
\text { age }\end{array}$ & $\begin{array}{l}\text { - } 1 \text { SD relative with br } \\
\text { ca } d x>\text { age } 50\end{array}$ \\
\hline
\end{tabular}

Personal history of ovarian cancer was not included because those with a previous diagnosis were not eligible for the study. If two SD relatives were involved, they had to be from the same side of the family.

br ca, breast cancer; dx, diagnosed; FD, first degree; hx, history; ov ca, ovarian cancer; SD, second degree.

aAll other respondents were classified as "average risk."

to be at "average" risk. These objective risk categories were based on US Preventive Services Task Force designations, other published risk stratification schemes, and expert opinion. ${ }^{8,9,11,26}$

Participants were also asked whether they had discussed their family history of cancer with a health-care provider (HCP), had been referred for genetic counseling for cancer risk, and had undergone $B R C A 1 / 2$ genetic testing.

To assess their perceived risk of ovarian cancer, women were asked whether their 10-year risk of developing ovarian cancer was much higher, higher, about the same, lower, or much lower than the risk of most women their age. They were also asked whether they thought their family history of cancer (any type) greatly increases, somewhat increases, has no effect, somewhat decreases, or greatly decreases their risk of cancer. Data collected on demographic characteristics included age, marital status, parity, race, education, and income level.

We obtained approval for this study from the institutional review boards of the Centers for Disease Control and Prevention 
and HFHS. We also obtained a Certificate of Confidentiality under section 301(d) of the Public Health Service Act given the sensitive information we were acquiring about personal and family history of cancer and genetic testing. All respondents gave consent before conducting the survey interviews.

\section{Statistical analyses}

We generated descriptive univariate statistics to evaluate the distribution of demographic characteristics (age, race, marital status, education level, income level, and parity) and other study variables, including risk group (average, elevated, and high), personal history of breast cancer, risk perception measures, and genetics-related health-care activities (having talked with an HCP regarding family cancer history, referral for genetic counseling, and undergoing genetic testing). In addition, we used Wald $\chi^{2}$ statistics to assess bivariate associations between the aforementioned variables and the outcomes of interest: genetic counseling and testing. Separate multivariable logistic regression models were used to obtain odds ratios (ORs) and 95\% confidence intervals (CIs) for genetic counseling and genetic testing, which were adjusted by the covariates. We used the Wald $\chi^{2}$ test to assess the associations between each outcome and the respective final model covariates, setting significance at $P \leq 0.05$. We also conducted a logistic regression analysis to obtain crude ORs and 95\% CIs for genetic counseling referral based on maternal versus paternal cancer family history. All estimates were weighted to account for differential selection probabilities, including the oversampling of women at high and elevated risk, and for nonresponse. We performed all statistical analyses using SAS 9.2 complex survey data procedures to account for the stratified sample design.

\section{RESULTS}

The distributions of demographic and other characteristics of participants, along with complete data for all study variables $(n=2,414)$, are given in Table 2 . The population was primarily white, married or partnered, well educated, and in the middle- to upper-income brackets. Most had no personal history of breast cancer, were in the average-risk category by family history, and were parous. A minority perceived themselves as being at increased risk for ovarian cancer, whereas many felt that their family history increased their cancer risks. With regard to genetics-related health-care activities, only a small percentage of participants had been referred for genetic counseling or had undergone genetic testing, whereas 30\% had discussed their family history of cancer with their HCP.

Figure 1 illustrates the relationship between the assigned risk categories and the genetics-related health-care activities in the entire sample. Participants in the high-risk category were significantly more likely to have talked with their HCP about their family history of cancer, been referred for cancer genetic counseling, and undergone $B R C A$ testing $(P<0.001$ for all comparisons). Similarly, those in the elevated-risk group were significantly more likely to have participated in these activities than those in the average-risk group $(P<0.001$ for all
Table 2 Distribution of demographic characteristics, cancer and perceived risk variables, and genetics-related health-care activities of 2,414 study participants

\begin{tabular}{|c|c|c|}
\hline Characteristic/variable & $n$ & Weighted (\%) \\
\hline \multicolumn{3}{|l|}{ Age (years) } \\
\hline$<40$ & 254 & 13.5 \\
\hline $41-50$ & 566 & 25.4 \\
\hline $51-60$ & 825 & 32.1 \\
\hline $61-64$ & 286 & 10.3 \\
\hline $65+$ & 483 & 18.7 \\
\hline \multicolumn{3}{|l|}{ Race } \\
\hline White & 1,623 & 66.0 \\
\hline Black & 653 & 28.0 \\
\hline Other & 138 & 6.0 \\
\hline \multicolumn{3}{|l|}{ Marital status } \\
\hline Married/partner & 1,621 & 67.6 \\
\hline Divorced/separated & 365 & 13.6 \\
\hline Single/never married & 222 & 10.7 \\
\hline Widowed & 206 & 8.1 \\
\hline \multicolumn{3}{|l|}{ Education level } \\
\hline$\leq$ High school/GED & 732 & 28.4 \\
\hline College, $<4$ years & 792 & 32.6 \\
\hline College, undergraduate degree & 474 & 20.8 \\
\hline Graduate degree & 416 & 18.2 \\
\hline \multicolumn{3}{|l|}{ Annual income $(\$)$} \\
\hline$<35,000$ & 505 & 20.2 \\
\hline $35,000-<50,000$ & 415 & 16.2 \\
\hline $50,000-<75,000$ & 568 & 23.5 \\
\hline$\geq 75,000$ & 926 & 40.2 \\
\hline \multicolumn{3}{|l|}{ Parity } \\
\hline Yes & 2,054 & 83.9 \\
\hline No & 360 & 16.1 \\
\hline \multicolumn{3}{|l|}{ Assigned risk category } \\
\hline High & 658 & 7.3 \\
\hline Elevated & 540 & 6.9 \\
\hline Average & 1,216 & 85.8 \\
\hline \multicolumn{3}{|l|}{ Personal history of breast cancer } \\
\hline No & 2,002 & 95.1 \\
\hline Yes, diagnosed at age $\leq 50$ years & 187 & 2.1 \\
\hline Yes, diagnosed at age $>50$ years & 225 & 2.8 \\
\hline \multicolumn{3}{|c|}{ Perceived 10-year risk of ovarian cancer } \\
\hline Lower & 934 & 46.0 \\
\hline Same & 1,010 & 41.1 \\
\hline Higher & 470 & 12.9 \\
\hline \multicolumn{3}{|c|}{ Perceived influence of family history on cancer risk } \\
\hline Decreases & 384 & 22.3 \\
\hline Has no effect & 749 & 33.3 \\
\hline Increases & 1,281 & 44.3 \\
\hline \multicolumn{3}{|c|}{ Talked to HCP about family cancer history } \\
\hline Yes & 520 & 29.9 \\
\hline No & 1,894 & 70.1 \\
\hline \multicolumn{3}{|l|}{ Referred for genetic counseling } \\
\hline Yes & 212 & 4.1 \\
\hline No & 2,202 & 95.9 \\
\hline \multicolumn{3}{|l|}{ Had $B R C A 1 / 2$ testing } \\
\hline Yes & 92 & 1.6 \\
\hline No & 2,322 & 98.4 \\
\hline
\end{tabular}

$\mathrm{GED}$, general educational development (high school equivalency); HCP, health-care provider.

aPercentages do not always sum to $100 \%$ because of rounding. 
ORIGINAL RESEARCH ARTICLE

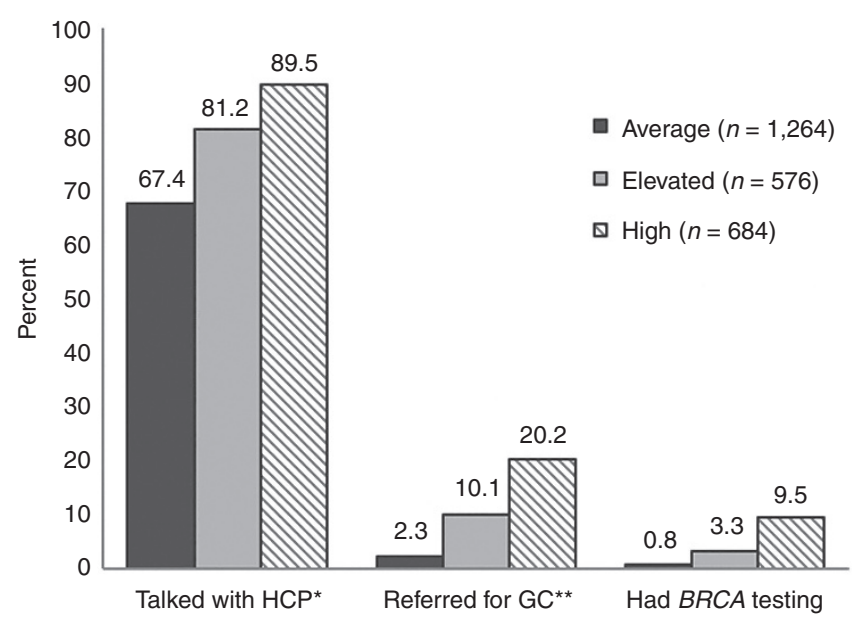

Figure 1 Genetics-related health-care activities by assigned risk category: discussion of family cancer history, referral for genetic counseling, and $\boldsymbol{B R C A}$ testing. *Talked with health-care provider about family cancer history. ${ }^{*}$ Genetic counseling. $P<0.001$ for all comparisons (average versus elevated versus high) for each activity.

comparisons). Nearly $90 \%$ of participants at high risk had discussed their family history of cancer with their HCP, although only $20 \%$ had actually been referred for genetic counseling services.

The associations between participant demographic characteristics along with other study variables, and individuals who had been referred for genetic counseling $(n=212)$ and those who had undergone $B R C A$ testing $(n=92)$, were also evaluated in bivariate analysis (Table 3). Patient age, race, and parity did not appear to be related to receipt of these services, whereas annual income, objective risk category, perceived 10-year risk of ovarian cancer, personal history of breast cancer, and having talked with an HCP about family cancer history were significantly associated with both genetic counseling referral and $B R C A$ testing. In addition, genetic counseling referral was highest for women with a graduate degree. Marital status, perceived influence of family history on risk, and having been referred for genetic counseling were all significantly associated with having undergone $B R C A$ testing.

When analyzed via multivariable analyses (Table 4), several participant characteristics continued to significantly influence whether the individual had been referred for genetic counseling $(n=212)$ or undergone BRCA genetic testing $(n=92)$. In terms of referral for genetic counseling, participants with graduate degrees were more than twice as likely to be referred for genetic counseling than those with less than or equal to a high school education (OR: 2.08; 95\% CI: 1.04-4.14). Women in the elevated- and high-risk categories were 2.23 (OR; 95\% CI: 1.41-3.54) and 5.74 (OR; 95\% CI: 3.82-8.64) times more likely to be referred than those at average risk. Participants who perceived their 10-year risk for ovarian cancer as higher than the general population also had a greater than twofold likelihood (OR: 2.14; 95\% CI: 1.34-3.43) of having been referred compared with those who perceived their risk to be the same or lower than that of the average woman. Personal history of breast cancer was associated with a more than threefold likelihood of referral. This effect was the same for both women diagnosed at 50 years of age or younger (OR: 3.10; $95 \% \mathrm{CI}$ : $2.27-4.22$ ) and those diagnosed after 50 years (OR: 3.12 ; $95 \%$ CI: $2.12-5.32$ ). Women who had spoken with their doctor about their family history reported referral to genetic counseling 2.4 times more often than those who had not (OR: 2.4; 95\% CI: 1.09-5.32).

Somewhat different patterns were seen among women who reported having undergone BRCA1/2 testing. Women who were single or had never married were $83 \%$ less likely to have undergone testing than those who were married or with a partner (OR: 0.17; 95\% CI: 0.07-0.44). Unlike having been referred, perceived risk because of family history strongly influenced whether a woman had undergone BRCA1/2 testing. Participants who felt that their family history had no effect on their risk were 4.6 (OR; 95\% CI: 2.20-9.64) times more likely to have undergone testing than those who reported a perceived decreased risk based on family history; whereas those who perceived that their family history increased their risk were more than 10 times as likely to report having undergone BRCA1/2 testing (OR: 10.44; 95\% CI: 3.89-27.99). Personal history of breast cancer was also associated with testing, with ORs of 8.05 (95\% CI: 4.99-13.00) and 5.33 (95\% CI: 2.20-9.64) for women diagnosed at or younger than 50 years of age and for those older than 50 years of age, respectively, compared with women with no personal history. Finally, women who had been referred for genetic counseling reported testing at a rate that was almost 13 times that of those who had not been referred (OR: 12.94; 95\% CI: 7.96-21.04).

Previous research has suggested that a paternal family history of breast and/or ovarian cancer is less likely to be identified as a reason for genetic counseling referral with regard to $B R C A 1 / 2$ testing. ${ }^{27}$ We also found a higher likelihood of being referred for counseling among women with a maternal versus paternal cancer family history, although this did not reach statistical significance (crude OR: 2.11; 95\% CI: 0.99-4.79).

\section{DISCUSSION}

Findings from this study offer unique insight into referral for genetic counseling and $B R C A$ testing of women from families that are at average, elevated, and high risk as classified based on detailed family history information. This large cohort of women is also unique because they received care within a system that provides both access to and coverage for genetic counseling and testing for HBOC.

Most women reported having talked with their HCP about their family cancer history, although this varied significantly by risk category, with almost $90 \%$ of those in the high-risk group having talked with their provider. By contrast, only $20 \%$ of women in the high-risk group reported having been referred for genetic counseling. This low referral percentage is particularly troubling because this health-care system provides both genetic counseling services and coverage for genetic testing. Barriers at the provider level might, in part, explain this finding. The 
Table 3 Relationships between demographic characteristics and other study variables and genetic counseling referral and $B R C A$ testing

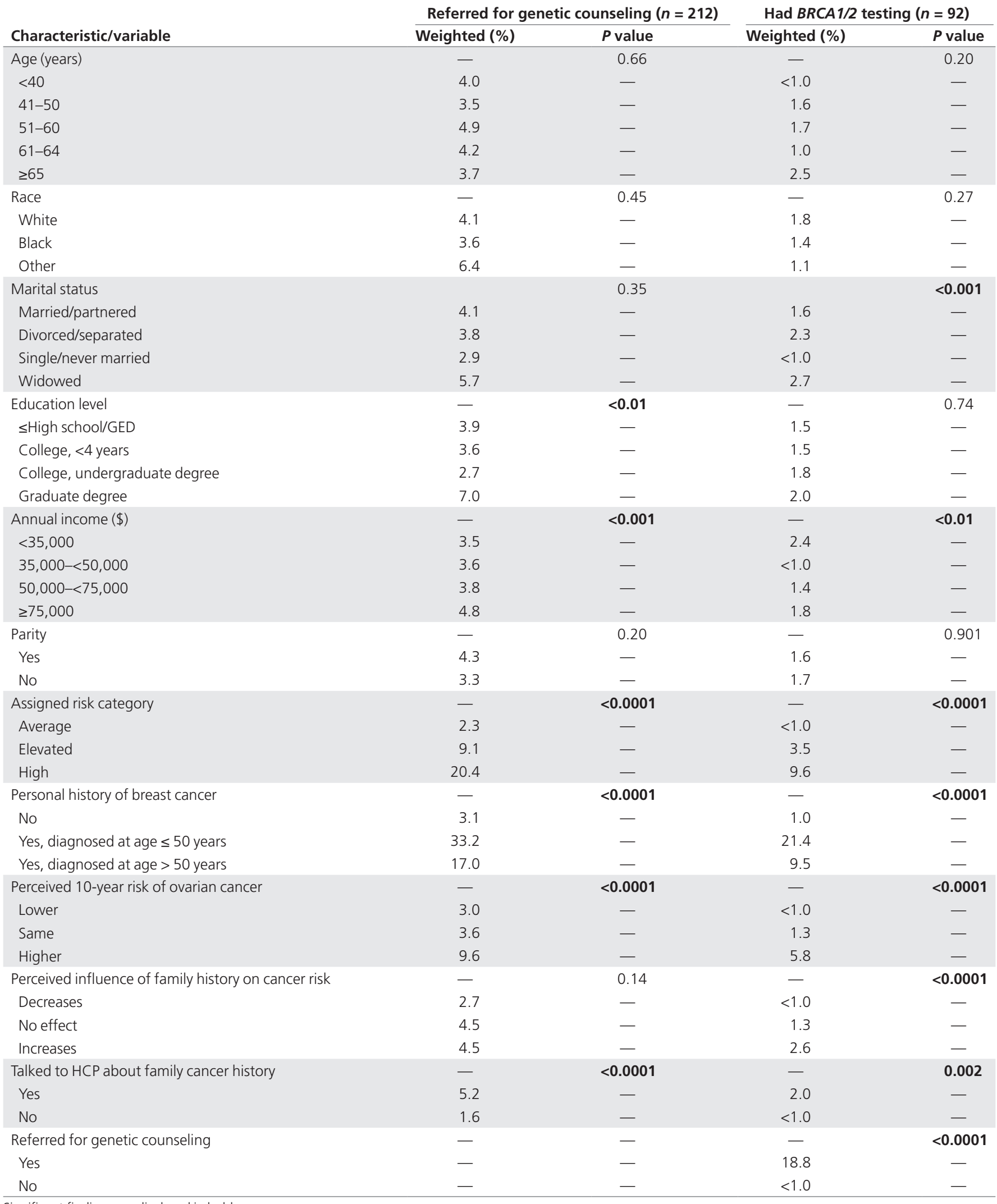

Significant findings are displayed in bold.

GED, general educational development (high school equivalency); HCP, health-care provider. 
Table 4 Adjusted odds ratios and $95 \%$ Cls for genetic counseling referral and $B R C A 1 / 2$ testing

\begin{tabular}{|c|c|c|}
\hline \multirow{2}{*}{$\begin{array}{l}\text { Participant } \\
\text { characteristic }\end{array}$} & $\begin{array}{l}\text { Referred for genetic } \\
\text { counseling }(n=212)\end{array}$ & $\begin{array}{l}\text { Had } B R C A 1 / 2 \\
\text { testing }(n=92)\end{array}$ \\
\hline & \multicolumn{2}{|c|}{ OR $(95 \% \mathrm{Cl})$} \\
\hline \multicolumn{3}{|l|}{ Age (years) } \\
\hline$<40$ & 1.00 & 1.0 \\
\hline $41-50$ & $0.61(0.31-1.23)$ & $1.30(0.34-4.86)$ \\
\hline $51-60$ & $0.80(0.37-1.76)$ & $1.13(0.29-4.30)$ \\
\hline $61-64$ & $0.67(0.26-1.73)$ & $0.82(0.23-2.90)$ \\
\hline$\geq 65$ & $0.53(0.21-1.35)$ & $2.50(0.48-13.13)$ \\
\hline \multicolumn{3}{|l|}{ Race } \\
\hline White & 1.00 & 1.00 \\
\hline Black & $1.07(0.63-1.81)$ & $1.36(0.57-3.25)$ \\
\hline Other & $1.92(0.84-4.41)$ & $0.55(0.29-1.03)$ \\
\hline \multicolumn{3}{|l|}{ Marital status } \\
\hline Married/partnered & 1.00 & 1.00 \\
\hline Divorced/separated & $0.79(0.46-1.37)$ & $1.18(0.52-2.69)$ \\
\hline Single/never married & $0.92(0.48-1.78)$ & $0.17(0.07-0.44)$ \\
\hline Widowed & $2.02(0.84-4.87)$ & $1.58(0.44-5.92)$ \\
\hline \multicolumn{3}{|l|}{ Education level } \\
\hline$\leq$ High school/GED & 1.00 & 1.00 \\
\hline College, $<4$ years & $0.87(0.49-1.53)$ & $1.27(0.39-4.20)$ \\
\hline College, & $0.65(0.37-1.15)$ & $1.87(0.40-8.68)$ \\
\hline
\end{tabular}

undergraduate

degree

Graduate degree

Annual income (\$)

$<35,000$

$2.08(1.04-4.14)$

$1.87(0.44-7.90)$

$35,000-<50,000$

1.00

$50,000-<75,000$

$0.99(0.50-1.98)$

$1.00(0.47-2.12)$

(0.10-0.62)

$\geq 75,000$

$1.17(0.58-2.34)$

Parity

No

1.00

$0.43(0.17-1.08)$

$0.48(0.12-1.81)$

Yes

Assigned risk category

Average

Elevated

(1.41-3.54)

High

$5.74(3.82-8.64)$

Personal history of breast cancer

No

1.00

Yes, diagnosed at

$3.10(2.27-4.22)$

$3.12(2.12-5.32)$

1.00

$1.77(0.49-6.82)$

age $\leq 50$ years

Yes, diagnosed at age $>50$ years

Perceived 10-year risk of ovarian cancer

Lower

1.00

Same

$1.01(0.59-1.76)$

Higher

$2.14(1.34-3.43)$

1.00

$0.67(0.28-1.63)$

$1.21(0.53-2.74)$

Perceived influence of family history on cancer risk

Decreases

1.00

No effect

$1.47(0.68-3.17)$

Increases

$1.00(0.50-2.01)$

Talked to HCP about family cancer history

$\begin{array}{lc}\text { No } & 1.00 \\ \text { Yes } & \mathbf{2 . 4 0}(\mathbf{1 . 0 9 - 5 . 3 2 )}\end{array}$

Referred for genetic counseling

No

-

1.00

$12.94(7.96-21.04)$

Significant findings are displayed in bold. All variables are included in multivariable logistic regression model.

$\mathrm{Cl}$, confidence interval; GED, general educational development (high school equivalency); $\mathrm{HCP}$, health-care provider; $\mathrm{OR}$, odds ratio. literature suggests that failure to identify and refer women at risk for HBOC may be ascribed to the lack of time to assess fully familial cancer history, ${ }^{28}$ lack of confidence in knowledge about genetic risk and referral guidelines, and medical specialty. ${ }^{29,30}$ Specific examples of the lack of knowledge about genetics include the failure to associate ovarian cancer with breast cancer risk and discounting paternal family history. ${ }^{27}$ Although not statistically significant, our data also suggested higher referral patterns among women with a family history of cancer in their maternal versus paternal lineage.

In assessing demographic characteristics that potentially influence genetic counseling and referral patterns, we found that higher income was not associated with either referral or testing when adjusted in multivariable analysis. These findings contradict other studies that suggest that patient income is a significant factor. ${ }^{29,31}$ Our findings might reflect the fact that our population tended to be more homogeneous, with higher levels of education and income than the general population. Furthermore, these women were in a health-care system with regular access to care, which is often not the case for low-income individuals. Similarly, education level was not highly predictive: only those with a graduate degree were more likely to receive a referral to genetic counseling. Other studies have suggested that both education level and knowledge of genetics are associated with endorsement of testing or uptake of genetic testing. ${ }^{32,33}$ Marital status had no effect on referral for genetic counseling and only a modest effect on BRCA testing, with those single or never married less likely to undergo testing. There was no relationship between parity and either referral to genetic counseling or testing. Race and age, noted in other studies to influence uptake of genetic services, ${ }^{34}$ also did not influence either referral or testing when adjusted for other factors. Although risk category strongly predicted referral, it did not appear to influence uptake of $B R C A$ testing, possibly because of the low numbers of women undergoing testing, particularly in the average- and elevated-risk groups. However, referral for genetic counseling was strongly associated (OR: 12.94) with undergoing BRCA testing, likely reflecting the protocols for genetic counseling and testing within the HFHS, where counseling is typically required for testing to be covered.

Previous data have suggested that younger women without breast cancer but with a positive family history express a greater interest in testing than do older women, whereas cancer survivors expressing an interest in testing tend to be older. ${ }^{19,21}$ Our data revealed that age impacted neither referral nor testing, although personal history of breast cancer impacted both. Although the age of onset of breast cancer ( $\leq 50$ years vs. $>50$ years) did not differ in terms of influence on referral for genetic counseling, it did strongly influence whether women underwent $B R C A$ testing (OR: 8.05 vs. 5.33 compared with women without a history of breast cancer). We suspect these differences may reflect the impact of the cancer genetic risk assessment and counseling process provided within the HFHS. 
We examined two risk perception factors in relation to referral for genetic counseling and having undergone $B R C A$ testing: perceived 10-year risk of ovarian cancer and perceived influence of one's family history on risk for cancer. Women with a higher perceived risk of developing ovarian cancer in the next 10 years were more than twice as likely to be referred for genetic counseling, even when adjusted for objective risk level and other variables, including personal history of breast cancer. We also found that a higher level of perceived risk of ovarian cancer is suggestive of undergoing $B R C A$ testing, although this association did not reach statistical significance. Nonetheless, participants who perceived that their family history increased their risk for cancer were substantially more likely to report having undergone BRCA testing than those who felt their family history indicated low risk. This is consistent with previous publications demonstrating that women with a strong family history of cancer $^{21,22,35}$ and heightened risk perception ${ }^{36}$ are more likely to express interest in or undergo genetic counseling and testing.

We must acknowledge several limitations of our study methodology and data. Most notably, our data rely on self-reported accounts of genetic counseling referral, BRCA1/2 testing, and family cancer history. To reduce the chances of misreporting, subjects were provided with detailed descriptions of these health-care activities as a part of the interview process. Because only 0.3 and $4 \%$ of survey respondents answered "don't know" to the questions regarding counseling referral and $B R C A$ testing, and no subject refused to answer, any bias would be expected to be minimal. With regard to cancer family history, previous studies suggest that the accuracy of reporting breast cancer in first-degree relatives is relatively high, although this diminishes in the case of second-degree relatives and appears to be less reliable for reporting ovarian cancer. ${ }^{37,38}$ In addition, our exclusion of women with a previous history of ovarian cancer or previous oophorectomy possibly led to an underestimate of the number of participants at high risk, although ovarian cancer survivors comprise less than $0.2 \%$ of women aged 30 years or older in the United States. ${ }^{39}$

Because there is no single standard for what constitutes "high" versus "elevated" versus "average" risk, our results should be considered in the context of our risk classification strategy and definition of terms; however, the resulting differences in referral and testing patterns suggest that our risk designations matched relatively closely with those determined by providers within the system. Notably, however, because we asked about referral for genetic counseling and not completion of genetic counseling, we are unable to address barriers that may prevent women who have been referred from actually seeking these services. Finally, our sample cannot be considered representative of the general population, although it is reflective of women within a healthcare system that provides access to genetics services.

\section{Conclusion}

Our results indicate that the availability of cancer genetics services does not, in and of itself, lead to appropriate utilization among women at high risk, in part because of underreferral for genetic counseling by system HCPs. Classic sociodemographic characteristics appear to have minimal impact on receiving a referral for genetic counseling and undergoing $B R C A$ testing in this setting of ready access, whereas objective risk category and perception of personal and familial cancer risk strongly influence these processes. Our study fills an important research gap identified in the recently published US Preventive Services Task Force updated recommendations with regard to $B R C A-$ namely the need to evaluate referral patterns, risk assessment, and $B R C A 1 / 2$ testing in settings other than high-risk centers. ${ }^{40}$ Further research is needed to evaluate patient decision making with regard to uptake of available genetic counseling and $B R C A 1 / 2$ testing services, and to evaluate interventions targeted toward improving HCP identification and referral of women at high risk.

\section{ACKNOWLEDGMENTS}

The findings and conclusions in this article are those of the authors and do not necessarily represent the official position of the Centers for Disease Control and Prevention. Funding support was provided by the Centers for Disease Control and Prevention (contract 200-2002-00574, task order 0015).

\section{DISCLOSURE}

The authors declare no conflict of interest.

\section{REFERENCES}

1. Risch HA, McLaughlin JR, Cole DE, et al. Population BRCA1 and BRCA2 mutation frequencies and cancer penetrances: a kin-cohort study in Ontario, Canada. J Nat/ Cancer Inst 2006;98:1694-1706.

2. Pal T, Permuth-Wey J, Betts JA, et al. BRCA1 and BRCA2 mutations account for a large proportion of ovarian carcinoma cases. Cancer 2005;104:28072816.

3. Nelson HD, Huffman LH, Fu R, Harris EL; U.S. Preventive Services Task Force. Genetic risk assessment and BRCA mutation testing for breast and ovarian cancer susceptibility: systematic evidence review for the U.S. Preventive Services Task Force. Ann Intern Med 2005;143:362-379.

4. Chen S, Parmigiani G. Meta-analysis of BRCA1 and BRCA2 penetrance. J Clin Oncol 2007;25:1329-1333.

5. Rebbeck TR, Kauff ND, Domchek SM. Meta-analysis of risk reduction estimates associated with risk-reducing salpingo-oophorectomy in BRCA1 or BRCA2 mutation carriers. J Nat/ Cancer Inst 2009;101:80-87.

6. Domchek SM, Friebel TM, Singer CF, et al. Association of risk-reducing surgery in BRCA1 or BRCA2 mutation carriers with cancer risk and mortality. JAMA 2010;304:967-975

7. Warner $E$, Hill K, Causer $P$, et al. Prospective study of breast cancer incidence in women with a BRCA1 or BRCA2 mutation under surveillance with and without magnetic resonance imaging. J Clin Onco/ 2011;29:1664-1669.

8. US Preventive Services Task Force. Genetic risk assessment and BRCA mutation testing for breast and ovarian cancer susceptibility: recommendation statement. Ann Intern Med 2005;143:355-361.

9. Lu K, Kauff N, Powell CB, et al. ACOG Practice Bulletin No. 103: hereditary breast and ovarian cancer syndrome. Obstet Gynecol 2009;113:957-966.

10. Berliner JL, Fay AM, Cummings SA, Burnett B, Tillmanns T. NSGC practice guideline: risk assessment and genetic counseling for hereditary breast and ovarian cancer. J Genet Couns 2013;22:155-163.

11. National Comprehensive Cancer Network. Clinical practice guidelines in oncology. Genetic/familial high-risk assessment: breast and ovarian. http:// www.nccn.org/professionals/physician_gls/pdf/genetics_screening.pdf. Accessed 1 March 2013

12. Wang G, Beattie MS, Ponce NA, Phillips KA. Eligibility criteria in private and public coverage policies for BRCA genetic testing and genetic counseling. Genet Med 2011;13:1045-1050. 


\section{ORIGINAL RESEARCH ARTICLE}

13. Narod SA, Foulkes WD. BRCA1 and BRCA2: 1994 and beyond. Nat Rev Cancer 2004;4:665-676.

14. Peters N, Rose A, Armstrong K. The association between race and attitudes about predictive genetic testing. Cancer Epidemiol Biomarkers Prev 2004;13:361-365.

15. Baer HJ, Brawarsky P, Murray MF, Haas JS. Familial risk of cancer and knowledge and use of genetic testing. J Gen Intern Med 2010;25:717-724.

16. Weitzel JN, Blazer KR, Macdonald DJ, Culver JO, Offit K. Genetics, genomics, and cancer risk assessment: State of the art and future directions in the era of personalized medicine. CA Cancer J Clin 2011; e-pub ahead of print 9 August 2011.

17. Forman $A D$, Hall MJ. Influence of race/ethnicity on genetic counseling and testing for hereditary breast and ovarian cancer. Breast J 2009;15(suppl 1):S56S62.

18. Kieran S, Loescher $\mathrm{LJ}$, Lim KH. The role of financial factors in acceptance of clinical BRCA genetic testing. Genet Test 2007;11:101-110.

19. Fraser L, Bramald S, Chapman C, et al. What motivates interest in attending a familial cancer genetics clinic? Fam Cancer 2003;2:159-168.

20. Van Asperen CJ, Van Dijk S, Zoeteweij MW, et al. What do women really want to know? Motives for attending familial breast cancer clinics. J Med Genet 2002;39:410-414.

21. Bottorff JL, Ratner PA, Balneaves LG, et al. Women's interest in genetic testing for breast cancer risk: the influence of sociodemographics and knowledge. Cancer Epidemiol Biomarkers Prev 2002;11:89-95.

22. Kessler L, Collier A, Brewster K, et al. Attitudes about genetic testing and genetic testing intentions in African American women at increased risk for hereditary breast cancer. Genet Med 2005;7:230-238.

23. Sivell S, Elwyn G, Gaff CL, et al. How risk is perceived, constructed and interpreted by clients in clinical genetics, and the effects on decision making: systematic review. J Genet Couns 2008;17:30-63.

24. Caruso A, Vigna C, Marozzo B, et al. Subjective versus objective risk in genetic counseling for hereditary breast and/or ovarian cancers. J Exp Clin Cancer Res 2009;28:157.

25. Leadbetter S, Hawkins NA, Scholl LE, et al. Recruiting women for a study on perceived risk of cancer: influence of survey topic salience and early versus late response. Prev Chronic Dis 2013;10:E75.

26. Lancaster JM, Powell CB, Kauff ND, et al.; Society of Gynecologic Oncologists Education Committee. Society of Gynecologic Oncologists Education Committee statement on risk assessment for inherited gynecologic cancer predispositions. Gynecol Oncol 2007;107:159-162.
BELLCROSS et al | Characteristics associated with utilization of BRCA genetics services

27. Burke W, Culver J, Pinsky L, et al. Genetic assessment of breast cancer risk in primary care practice. Am J Med Genet A 2009;149A:349-356.

28. Rich EC, Burke W, Heaton CJ, et al. Reconsidering the family history in primary care. J Gen Intern Med 2004;19:273-280.

29. Bellcross CA, Kolor K, Goddard KA, Coates RJ, Reyes M, Khoury MJ. Awareness and utilization of $B R C A 1 / 2$ testing among U.S. primary care physicians. Am J Prev Med 2011;40:61-66.

30. Sabatino SA, McCarthy EP, Phillips RS, Burns RB. Breast cancer risk assessment and management in primary care: provider attitudes, practices, and barriers. Cancer Detect Prev 2007;31:375-383.

31. Kieran S, Loescher LJ, Lim KH. The role of financial factors in acceptance of clinical BRCA genetic testing. Genet Test 2007;11:101-110.

32. Peters N, Domchek SM, Rose A, Polis R, Stopfer J, Armstrong K. Knowledge, attitudes, and utilization of BRCA1/2 testing among women with early-onset breast cancer. Genet Test 2005;9:48-53.

33. Simon MS, Petrucelli N. Hereditary breast and ovarian cancer syndrome: the impact of race on uptake of genetic counseling and testing. Methods $\mathrm{Mol} B \mathrm{BiO}$ 2009;471:487-500.

34. Armstrong K, Micco E, Carney A, Stopfer J, Putt M. Racial differences in the use of BRCA $1 / 2$ testing among women with a family history of breast or ovarian cancer. JAMA 2005;293:1729-1736.

35. Bellcross CA, Leadbetter S, Alford SH, Peipins LA. Prevalence and healthcare actions of women in a large health system with a family history meeting the 2005 USPSTF recommendation for BRCA genetic counseling referral. Cancer Epidemiol Biomarkers Prev 2013;22:728-735.

36. Shiloh S, Ilan S. To test or not to test? Moderators of the relationship between risk perceptions and interest in predictive genetic testing. J Behav Med 2005;28:467-479.

37. Mai PL, Garceau AO, Graubard BI, et al. Confirmation of family cancer history reported in a population-based survey. J Natl Cancer Inst 2011;103: 788-797.

38. Murff HJ, Spigel DR, Syngal S. Does this patient have a family history of cancer? An evidence-based analysis of the accuracy of family cancer history. JAMA 2004;292:1480-1489.

39. Cancer survivors - United States, 2007. Morb Mort Wkly Rep 2011;60:269272.

40. Moyer VA. Risk assessment, genetic counseling, and genetic testing for BRCArelated cancer in women: U.S. Preventive Services Task Force Recommendation Statement. Ann Intern Med 2014;160:271-281. 